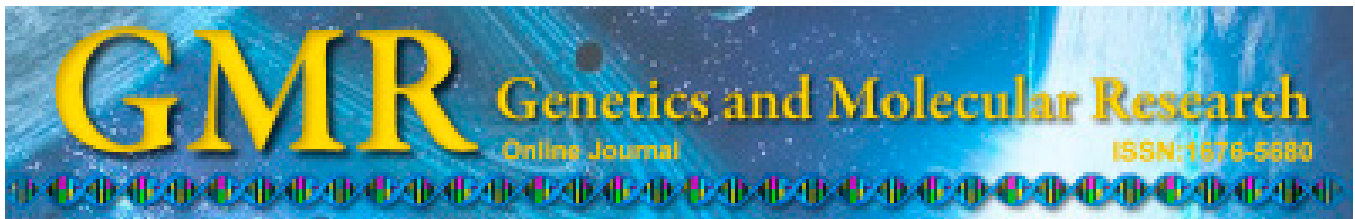

\title{
Correlation between the decrease of cholesterol efflux from macrophages in patients with type II diabetes mellitus and down-regulated CYP7A1 expression
}

\author{
L.D. Bao ${ }^{1 *}$, C.Q. Li ${ }^{2 *}$, R. Peng ${ }^{1}$, X.H. Ren ${ }^{1}$, R.L. Ma ${ }^{1}$, Y. Wang ${ }^{1}$ and H.J. Lv $^{3}$ \\ ${ }^{1}$ Department of Pharmacy, \\ Affiliated Hospital of Inner Mongolia Medical University, Hohhot, China \\ ${ }^{2}$ Department of Geriatrics, \\ Affiliated Hospital of Inner Mongolia Medical University, Hohhot, China \\ ${ }^{3}$ Department of Scientific Research, \\ Affiliated Hospital of Inner Mongolia Medical University, Hohhot, China \\ *These authors contributed equally to this study. \\ Corresponding authors: H.J. Lv / Y. Wang \\ E-mail: 1vhaijundsr@163.com / wang_yi237@163.com
}

Genet. Mol. Res. 14 (3): 8716-8724 (2015)

Received November 3, 2014

Accepted April 27, 2015

Published July 31, 2015

DOI http://dx.doi.org/10.4238/2015.July.31.20

\begin{abstract}
The purpose of this study was to examine the changes of cellular cholesterol efflux from macrophages in patients with type II diabetes mellitus (DM), and to determine the expression of CYP7A1, ABCG5, and LXR $\beta$ therein. We recruited 30 patients with type II DM (including 15 patients complicated with coronary heart disease and 15 patients with DM only) and 15 normal controls for this study. Peripheral blood monocytes were isolated for macrophage culture. The mRNA and protein expression levels of CYP7A1, ABCG5, and LXR $\beta$ were determined using real-time polymerase chain reaction and western blot. The macrophage cholesterol efflux rate was determined with $10 \%$ autoserum and standard serum as receptors. We determined
\end{abstract}


that the expression levels of macrophage CYP7A1 mRNA and protein in the type II DM group were significantly lower than those in the control group, but no differences were found in the ABCG5 and LXR $\beta$ expression levels between the groups. The macrophage cholesterol efflux rate in the patients with type II DM was also significantly decreased compared with that of the normal control subjects $(\mathrm{P}<0.01)$. Furthermore, CYP7A1 mRNA expression and macrophage cholesterol efflux rate were significantly positively correlated. In summary, this study demonstrated that the macrophage cholesterol efflux in patients with type II DM was significantly reduced, and that this reduction was associated with the down-regulation of CYP7A1 expression.

Key words: Diabetes mellitus; Monocyte macrophage; CYP7A1; Cholesterol efflux; ABCG5; LXR $\beta$

\section{INTRODUCTION}

Diabetes mellitus (DM) is a group of endocrine and metabolic diseases with unknown pathogenesis and primarily characterized by hyperglycemia and glycosuria. DM is generally a consequence of disorders of glucose, protein and lipid metabolism caused by absolute or relative deficiencies in blood insulin, and manifests as polydipsia, polyuria, polyphagia, and emaciation in the clinic (dos Santos-Weiss et al., 2012). DM is classified into primary and secondary categories, with the former being the most often referenced. DM can also be classified into two types according to its onset characteristics, i.e., type I and type II DM. Previous studies have found that patients with type II DM commonly suffer from abnormal cholesterol efflux, which is involved in the accumulation of cellular cholesterol and the progression of atherosclerosis (Penfornis et al., 2012; Dubé et al., 2013). However, the mechanism for the cholesterol efflux dysfunction in patients with type II DM remains undefined. This study aimed to explore the changes of cellular cholesterol efflux in patients with type II DM and the expression of receptors mediating cholesterol efflux utilizing primary cultured mononuclear macrophages as the source material.

\section{MATERIAL AND METHODS}

\section{Reagents}

The reagents in this study include: human lymphocyte separating medium, obtained from the Institute of Biomedical Engineering, Chinese Academy of Medical Sciences (Beijing, China); mouse anti-CYP7A1 polyclonal antibody, rabbit anti-human LXR $\beta$ polyclonal antibody, and rabbit anti-human ABCG5 polyclonal antibody (Fermentas, Hanover, MD, USA); a human peripheral blood CD14 cell sorting kit (Hangzhou Biowish Biotech Co., Ltd., Hangzhou, China); a Super-Script III First-Strand Synthesis System and Power SYBR Green premixed kit (Invitrogen, SantaCruz, CA, USA); and $\left[{ }^{3} \mathrm{H}\right]$-cholesterol oleate, obtained from the Beijing Kangpuhuiwei Technology Co., Ltd., Beijing, China. 


\section{Cell culture}

Peripheral blood $(20 \mathrm{~mL})$ was drawn from all subjects who met the diagnostic criteria $12 \mathrm{~h}$ after fasting for peripheral monocytes separation with human lymphocyte separating medium $(\mathrm{d}=1.126)$, and then monocytes were obtained using the human peripheral blood CD14 cell sorting kit, and seeded on 6-well plates $\left(1.0 \times 10^{5} /\right.$ well $)$ and 24 -well plates $\left(1.0 \times 10^{4} /\right.$ well), to which $10 \%$ autoserum $+20 \mathrm{ng} / \mathrm{mL}$ macrophage colony-stimulating factor (M-CSF) + RPMI1640 was added. Cells were cultured for $48 \mathrm{~h}$ to allow for their differentiation into macrophages, which were subsequently identified by oil red $\mathrm{O}$ staining and CD68 immunohistochemical staining methods.

\section{Real-time polymerase chain reaction (PCR) detection of $C Y P 7 A 1, A B C G 5$, and LXR $\beta$ mRNA levels}

Total cellular RNA was routinely extracted with TRIzol. The sample RNA purity $($ A260/A280 $=1.70-1.90)$ and content were detected using DU-800 nucleic acid ultraviolet analyzer (Beckman, CA, USA). The Super-Script III First-Strand Synthesis System was used for reverse transcription to obtain the first-strand cDNA.

The real-time PCR conditions were as follows: detection of $A B C G 5$ and $L X R \beta$ was performed by the standard two-step method, with an initial denaturation at $92^{\circ} \mathrm{C}$ for $30 \mathrm{~s}$, denaturation at $92^{\circ} \mathrm{C}$ for $20 \mathrm{~s}$, and extension at $70^{\circ} \mathrm{C}$ for $30 \mathrm{~s}$ for a total of 30 cycles; CYP7A1 detection was conducted by the three-step method: initial denaturation at $92^{\circ} \mathrm{C}$ for $30 \mathrm{~s}$, denaturation at $92^{\circ} \mathrm{C}$ for $20 \mathrm{~s}$, annealing at $45^{\circ} \mathrm{C}$ for $10 \mathrm{~s}$, and extension at $70^{\circ} \mathrm{C}$ for $30 \mathrm{~s}$ for a total of 30 cycles. The difference in the template quantity was corrected with GAPDH as the reference gene. The PCR was conducted three separate times for each sample, with triplicate wells per each PCR; those three sets of triplicate wells were used during a single PCR. The primer sequences for $C Y P 7 A 1$ were as follows: upstream 5'-AGA GAT TTA CTG GCA GCT GC-3', downstream 5'-ACA CCA CCA GAT ACA GAA GG-3'; the primer sequences for $A B C G 5$ were: upstream 5'-GCG ACA CTT CAA CCA CAC CAT-3', downstream 5'-AGC CAA GAC ACA GAT ACC AG-3'; the primer sequences for $L X R \beta$ were: upstream 5'-CAG GAA TTC GGC GGA ACT TT-3', downstream 5'-CAT AAA ATT CCA GGC CCG TTG T-3'; and the primer sequences for GAPDH were: upstream 5'-TAC ATT CTA TGC ATG GTG TC3', downstream 5'- CGC ATC ACG ATT CTC CTC AC-3'. The gene expression levels of each sample and the reference sample were calculated using the 7500 System SDS software (ABI, USA) (Kojima et al., 2009).

\section{Western blot detection of CYP7A1, ABCG5, and LXRß protein levels}

After cell lysis, a $50-\mu \mathrm{g}$ protein sample aliquot was mixed with a sodium dodecyl sulfate (SDS) buffer solution, which was separated by SDS-polyacrylamide gel electrophoresis (SDS-PAGE) and electro-transferred to a nitrocellulose membrane, blocked with $10 \%$ skimmed milk powders for $3 \mathrm{~h}$, and then incubated with mouse anti-CYP7A1 (1:500), rabbit anti-ABCG5 (1:1000), or rabbit anti-LXR $\beta(1: 1000)$ respectively, at $4{ }^{\circ} \mathrm{C}$ overnight. After addition of the secondary antibody, the membranes were incubated at room temperature for 2 $\mathrm{h}$, and washed with phosphate buffered saline/Tween 20 (PBST) for color development using 
the Super Signal West Pico chemiluminescence system (Snibe, Shenzhen, China). The protein levels were expressed by the ratio of the absorbance of the target gene by IMMAGE 800 Protein Analysis System (Beckman Coulter, NY, USA).

\section{Determination of the cholesterol efflux rate}

After the monocytes had been cultured for $48 \mathrm{~h}$ to permit successful differentiation into macrophages, they were supplemented with $2.0 \times 10^{4} \mathrm{~Bq} / \mathrm{mL}\left[{ }^{3} \mathrm{H}\right]$-cholesterol incubation medium and incubated at $37^{\circ} \mathrm{C}$ for $12 \mathrm{~h}$, and then cholesterol efflux was induced by $10 \%$ autoserum and standard serum. A Beckman liquid scintillation counter (Beckman Coulter, Brea, CA, USA) was used for quantification. The cholesterol efflux rate was calculated as the efflux liquid count / (efflux liquid count + lysis buffer count) x 100\%.

\section{Statistical analysis}

All data are reported as means \pm standard deviation, and statistical analysis was performed using the SPSS 13.0 software (SPSS, Chicago, IL, USA). One-way ANOVA was used for intra-group comparison and Pearson's bivariate analysis was used for correlation analysis.

\section{RESULTS}

\section{Basic information}

A total of 60 patients with type II DM were selected and divided into two subgroups: a DM group $(\mathrm{N}=30)$ and a $\mathrm{DM}$ complicated with coronary heart disease (CHD) group $(\mathrm{N}=30)$. The normal control group consisted of 30 subjects with matched ages and genders. The basic characteristics and biochemical indexes of the patients are shown in Table 1.

\begin{tabular}{|c|c|c|c|}
\hline & Control & DM & DM complicated with CHD \\
\hline Age & $48.6 \pm 11.5$ & $49.2 \pm 12.4$ & $48.9 \pm 11.8$ \\
\hline $\mathrm{M} / \mathrm{F}(\%)$ & $17(56.67 \%)$ & $16(53.33 \%)$ & $19(63.33 \%)$ \\
\hline BMI & $28.6 \pm 3.2$ & $29.3 \pm 2.8$ & $28.8 \pm 2.7$ \\
\hline FBG & $6.2 \pm 1.1$ & $9.4 \pm 1.6^{*}$ & $8.7 \pm 1.3$ \\
\hline $\mathrm{HbA1c}$ & - & $9.1 \pm 1.4$ & $9.2 \pm 1.3$ \\
\hline TC & $6.1 \pm 1.1$ & $5.8 \pm 1.2$ & $5.7 \pm 1.0$ \\
\hline TG & $2.1 \pm 0.4$ & $2.2 \pm 0.5$ & $2.1 \pm 0.5$ \\
\hline HDL-C & $1.8 \pm 0.5$ & $0.9 \pm 0.3^{*}$ & $1.7 \pm 0.4$ \\
\hline LDL-C & $2.9 \pm 0.7$ & $2.7 \pm 0.5$ & $2.8 \pm 0.6$ \\
\hline Hs-CRP & $1.6 \pm 0.6$ & $4.7 \pm 1.2 *$ & $5.0 \pm 1.3^{*}$ \\
\hline Smoking history [N (\%)] & $7(23.33 \%)$ & $8(26.67 \%)$ & $6(20.00 \%)$ \\
\hline
\end{tabular}

$\mathrm{DM}=$ type $\mathrm{II}$ diabetes mellitus; $\mathrm{CHD}=$ coronary heart disease; $\mathrm{BMI}=$ body mass index; $\mathrm{FBG}=$ fasting blood glucose; HbA1c = hemoglobin A1c; TC = total white blood cell count; $\mathrm{TG}=$ triglycerides; HDL-C = high density lipoprotein-cholesterol; LDL-C = low density lipoprotein-cholesterol; Hs-CRP = high sensitivity C-reactive protein. Compared with the control group, $* \mathrm{P}<0.05$. 


\section{mRNA expression of $C Y P 7 A 1, A B C G 5$, and $L X R \beta$ in monocyte macrophages}

The CYP7A1 mRNA expression level in monocyte macrophages from the type II DM group was significantly lower than that in control group $(\mathrm{P}<0.01)$, while no significant difference was found between the two type II DM subgroups. There were no significant differences in the expression levels of $A B C G 5$ and $L X R \beta$ mRNA between the groups (Table 2).

Table 2. mRNA expression of $C Y P 7 A 1, A B C G 5$, and $L X R \beta$ in the two groups.

\begin{tabular}{lccc}
\hline & Control & DM & DM complicated with CHD \\
\hline CYP7Al & $3.3 \pm 0.7$ & $1.0 \pm 0.6^{*}$ & $1.1 \pm 0.7^{*}$ \\
$A B C G 5$ & $4.2 \pm 1.0$ & $4.4 \pm 1.1$ & $4.3 \pm 1.0$ \\
LXR3 & $1.3 \pm 0.2$ & $1.4 \pm 0.3$ & $1.5 \pm 0.3$ \\
\hline
\end{tabular}

$\mathrm{DM}=$ type II diabetes mellitus; $\mathrm{CHD}=$ coronary heart disease. Compared with the control group, $* \mathrm{P}<0.05$.

\section{Protein expression of CYP7A1, ABCG5, and LXRß in monocyte macrophages}

The CYP7A1 protein expression level in the mononuclear macrophages was significantly decreased in the type II DM patient group compared to that in the normal control group $(\mathrm{P}<0.05)$, while there were no significant differences in the ABCG5 and LXR $\beta$ protein expression levels between the groups (Figure 1).
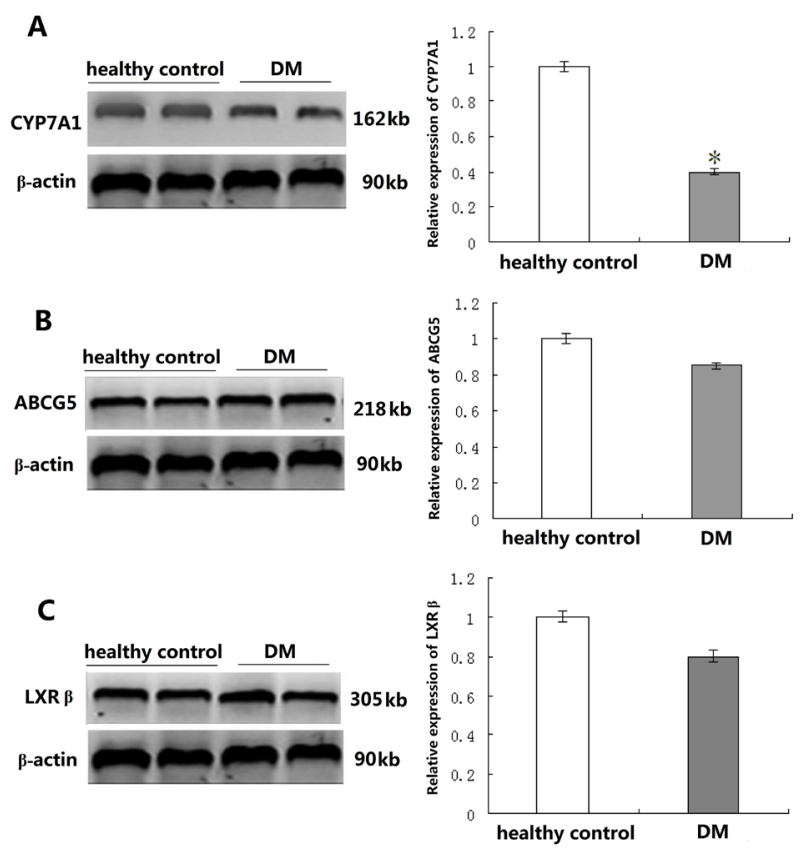

Figure 1. Protein expression of CYP7A1, ABCG5, and LXR $\beta$. A. Protein expression of CYP7A1 as determined by western blot. B. Protein expression of ABCG5; C. protein expression of LXR $\beta$. Compared with the control group, ${ }^{*} \mathrm{P}<0.01$. 


\section{Cholesterol efflux of monocyte macrophages}

The standard serum and autoserum-induced cholesterol efflux levels in patients with type II DM were significantly lower than those in normal control subjects $(\mathrm{P}<0.01)$, while no significant difference was found among the type II DM subgroups (Figure 2). In addition, the level of autoserum-induced cholesterol efflux was further reduced compared with the level of standard serum-induced cholesterol efflux in the type II DM group $(\mathrm{P}<0.01)$. There were no significant differences between standard serum and autoserum-induced cholesterol efflux levels in the normal control group (Figure 3).
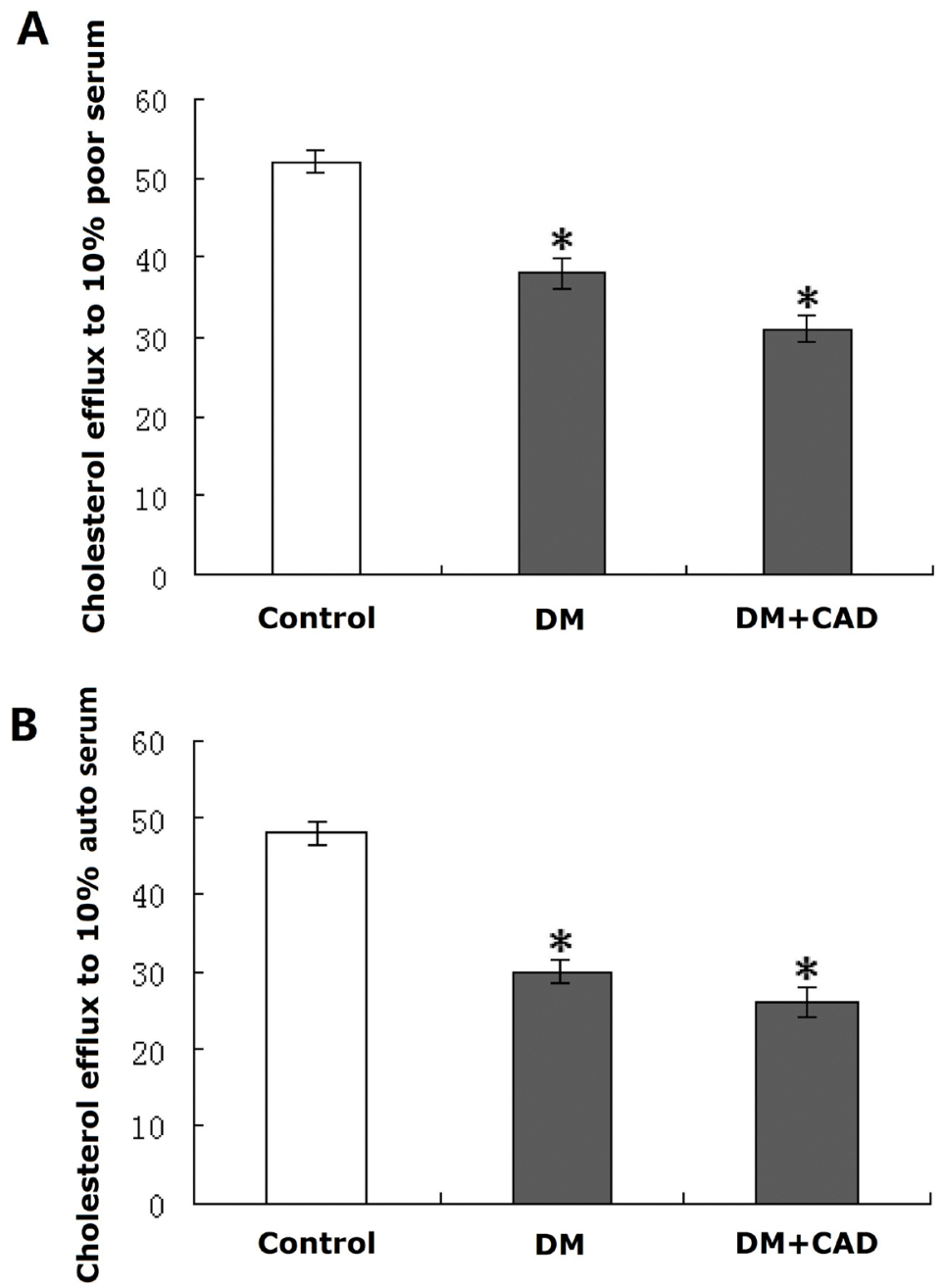

Figure 2. Cholesterol efflux rates induced by $10 \%$ standard serum and autoserum in macrophages. A. $10 \%$ standard serum-induced cholesterol efflux rate; B. autoserum-induced cholesterol efflux rate. Compared with the control group, $* \mathrm{P}<0.01 . \mathrm{DM}=$ type $\mathrm{II}$ diabetes mellitus; $\mathrm{CAD}=$ coronary artery disease. 


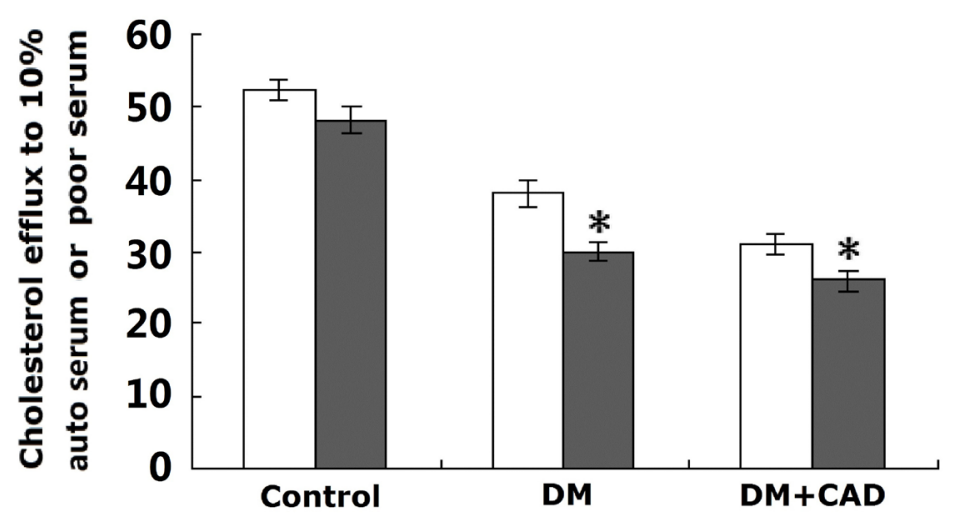

Figure 3. Comparison between $10 \%$ standard serum- (A) and autoserum-induced (B) cholesterol efflux rates. Comparison between one group, ${ }^{*} \mathrm{P}<0.01$. $\mathrm{DM}=$ type II diabetes mellitus; $\mathrm{CAD}=$ coronary artery disease.

\section{Correlation between $C Y P 7 A 1 \mathrm{mRNA}$ expression level and cholesterol efflux rate}

The CYP7A1 mRNA level was positively correlated with the $10 \%$ autoserum and standard serum-induced cholesterol efflux rates, and the correlation coefficients were $r=0.681$ and $\mathrm{r}=0.723(\mathrm{P}<0.01)$.

\section{DISCUSSION}

To date, the conclusions of studies on the expression and function of CYP7A1 in patients with type II DM and in associated animal models have been inconsistent (Shin and Osborne, 2008; Espinoza-Jiménez et al., 2012). The results of this study did not find abnormal CYP7A1 expression in the macrophages of patients with type II DM. The reasons for the prior inconsistent study results might include the following aspects: 1) a difference in the selection of animal species and cell lines leading to differences in gene expression; 2) individual differences between subjects, and 3) the functional impairment of CYP7A1 in some patients might be effected instead by post-translational regulation (Huang et al., 2011; Flaquer et al., 2012; Liu et al., 2012). Furthermore, some researchers have found a reduction of CYP7A1 expression in patients with hypertriglyceridemia, but no difference was found in the blood lipid levels among the patients in each group in this study, which might be the reason for the inconsistency between our results and those mentioned above (Dinneen and O'Donnell, 2012; Pérez de Isla et al., 2012). Both in vitro and in vivo studies have shown that CYP7A1 receptor regulation plays a major role in macrophage cholesterol efflux, accounting for about 60 to $70 \%$ of the total cholesterol efflux, whereas ABCG5 and LXR $\beta$ have little effect on this process (Brindisi et al., 2012; Querton et al., 2012). This study also found that LXR $\beta$ exhibited low expression in human monocyte-derived macrophages, and no significant difference was found in the expression levels among the groups, which is consistent with previous studies.

The mechanism involved in macrophage cholesterol efflux primarily includes receptor-mediated and watery diffusion pathways, in which CYP7A1, LXR $\beta$, and ABCG5 com- 
prise the major receptors mediating cholesterol efflux from macrophages (Malave et al., 2012; Oliveira et al., 2012). The efflux of cholesterol from macrophage foam cells on the vessel wall, as the primary step of reverse cholesterol transport, plays an extremely important part in preventing lipid deposition on the vascular wall and the progression of atherosclerosis (Low et al., 2012; Marrs, 2012). Studies have shown that type II DM might affect the function and regulation of the cholesterol transport receptor, thus causing the abnormal cholesterol efflux from macrophages (Ganda et al., 2012; Yoshida et al., 2012). In this study, macrophages differentiated from human peripheral blood monocytes were taken as the cells used for analysis, and our results demonstrated that the mRNA and protein expression levels of CYP7A1 on the macrophage surface in patients with type II DM were significantly lower than those in normal control subjects, whereas no significant difference was found among the type II DM subgroups. Consistent with this, our results also demonstrated that the serum-mediated cholesterol efflux rate was significantly decreased in patients with type II DM as well. These results suggest that the reduction of CYP7A1 expression in the macrophages of patients with type II DM is likely to play a key role in the formation of foam cells in diabetic vascular lesions, which is consistent with the results of recent animal experiments and in vitro studies.

Our study also found that the efflux function of cholesterol from macrophages was significantly impaired in patients with type II DM, consistent with the decreased expression of CYP7A1. These two findings were significantly correlated, which suggested that the functional impairment of cholesterol efflux in patients with type II DM might be closely associated with the reduction of $C Y P 7 A 1$ expression. In addition, we also compared the cholesterol effluxes mediated by standard serum and autoserum, and the results show that in patients with type II $\mathrm{DM}$, autoserum-mediated cholesterol efflux was further decreased compared with the standard serum-mediated cholesterol efflux. Some researchers have found that the cholesterol efflux from HepG2 cells induced by HDL particles was significantly reduced in patients with type II $\mathrm{DM}$, indicating that there might also be an certain degree of abnormality in the apolipoprotein and serum components in type II DM patients, which could also contribute to the abnormal impairment of cholesterol efflux (Wang et al., 2011).

Overall, this study demonstrated that the cholesterol efflux from peripheral blood monocyte-derived macrophages in patients with type II DM was significantly impaired, and that the mRNA and protein expression levels of CYP7A1 were significantly reduced. These two findings were shown to be closely correlated, which suggests that together they play an important role in the progression of atherosclerosis in patients with type II DM.

\section{Conflicts of interest}

The authors declare no conflict of interest.

\section{ACKNOWLEDGMENTS}

Research supported by the Nature Science Foundation of Inner Mongolia Autonomous Region (\#2013MS1224), a Scientific Project of the Affiliated Hospital of Inner Mongolia Medical University (\#NYFY2010YB006), the Youth Innovation Fund of Inner Mongolia Medical University (\#NY2010QN002), and the Key Scientific Fund of the Affiliated Hospital of Inner Mongolia Medical University (\#NYFYZD20130158). 


\section{REFERENCES}

Brindisi MC, Guiu B, Duvillard L, Athias A, et al. (2012). Liver fat content is associated with an increase in cholesterol synthesis independent of statin therapy use in patients with type 2 diabetes. Atherosclerosis 224: 465-468.

Dinneen SF and O'Donnell M (2012). ACP Journal Club. Review: quality improvement strategies reduce HbA(1c), LDL cholesterol, and BP in diabetes. Ann. Intern. Med. 157: JC4-11.

dos Santos-Weiss IC, Réa RR, Fadel-Pincheth CM, Rego FG, et al. (2012). The plasma logarithm of the triglyceride/ HDL-cholesterol ratio is a predictor of low risk gestational diabetes in early pregnancy. Clin. Chim. Acta 418: 1-4.

Dubé E, Ethier-Chiasson M and Lafonde J (2013). Modulation of cholesterol transport by insulin-treated gestational diabetes mellitus in human full-term placenta. Biol. Reprod. 88: 16.

Espinoza-Jiménez A, Peón, AN and Terrazas LI (2012). Alternatively activated macrophages in types 1 and 2 diabetes. Mediators Inflamm. 2012: 815953.

Flaquer M, Franquesa M, Vidal A, Bolaños N, et al. (2012). Hepatocyte growth factor gene therapy enhances infiltration of macrophages and may induce kidney repair in $\mathrm{db} / \mathrm{db}$ mice as a model of diabetes. Diabetologia 55: 2059-2068.

Ganda OP, Jumes CG, Abrahamson MJ and Molla M (2012). Quantification of concordance and discordance between apolipoprotein-B and the currently recommended non-HDL-cholesterol goals for cardiovascular risk assessment in patients with diabetes and hypertriglyceridemia. Diabetes Res. Clin. Pract. 97: 51-56.

Huang Q, An Y, Tang L, Jiang X, et al. (2011). A dual enzymatic-biosensor for simultaneous determination of glucose and cholesterol in serum and peritoneal macrophages of diabetic mice: evaluation of the diabetes-accelerated atherosclerosis risk. Anal. Chim. Acta 707: 135-141.

Kojima M, Ashino T, Yoshido T, Iwakura Y, et al. (2009). IL-1 regulates the Cyp7al gene and serum total cholesterol level at steady state in mice. Biochem. Biophys. Res. Commun. 379: 239-242.

Liu HF, Zhang HJ, Hu QX, Liu XY, et al. (2012). Altered polarization, morphology, and impaired innate immunity germane to resident peritoneal macrophages in mice with long-term type 2 diabetes. J. Biomed. Biotechnol. 2012: 867023.

Low H, Hoang A, Forbes J, Thomas M, et al. (2012). Advanced glycation end-products (AGEs) and functionality of reverse cholesterol transport in patients with type 2 diabetes and in mouse models. Diabetologia 55: 2513-2521.

Malave H, Castro M, Burkle J, Voros S, et al. (2012). Evaluation of low-density lipoprotein particle number distribution in patients with type 2 diabetes mellitus with low-density lipoprotein cholesterol $<50 \mathrm{mg} / \mathrm{dL}$ and non-high-density lipoprotein cholesterol <80 mg/dL. Am. J. Cardiol. 110: 662-665.

Marrs JC (2012). Glucose and low-density lipoprotein cholesterol lowering in elderly patients with type 2 diabetes: focus on combination therapy with colesevelam HCl. Drugs Aging 29: e1-e12.

Oliveira CP, Maranhao RC, Bertato MP, Wajchenberg BL, et al. (2012). Removal from the plasma of the free and esterified forms of cholesterol and transfer of lipids to HDL in type 2 diabetes mellitus patients. Lipids Health Dis. 11: 65.

Penfornis A, Baleydier A, Clavel T and Picard S (2012). LDL-cholesterol target values and actual values in patients with type 2 diabetes (T2D) uncontrolled on oral antidiabetic monotherapy: the lipid results of the French ESCALADE survey. Ann. Endocrinol. 73: 503-509.

Pérez de Isla L, Saltijeral Cerezo A, Vitale G, Gonzáles Timón B, et al. (2012). Prevalence of inapropriate LDL cholesterol levels in patients with coronary disease and/or type 2 diabetes. Rev. Clin. Esp. 212: 475-481.

Querton L, Buysschaert M and Hermans MP (2012). Hypertriglyceridemia and residual dyslipidemia in statin-treated, patients with diabetes at the highest risk for cardiovascular disease and achieving very-low low-density lipoproteincholesterol levels. J. Clin. Lipidol. 6: 434-442.

Shin DJ and Osborne TF (2008). Peroxisome proliferator-activated receptor-gamma coactivator-1alpha activation of CYP7A1 during food restriction and diabetes is still inhibited by small heterodimer partner. J. Biol. Chem. 283: 15089-15096.

Wang Y, Snel M, Jonker JT, Hammer S, et al. (2011). Prolonged caloric restriction in obese patients with type 2 diabetes mellitus decreases plasma CETP and increases apolipoprotein AI levels without improving the cholesterol efflux properties of HDL. Diabetes Care 34: 2576-2580.

Yoshida H, Hirowatari Y, Kurosawa H, Manita D, et al. (2012). Estimation of lipoprotein profile in patients with type II diabetes and its relevance to remnant lipoprotein cholesterol levels. Atherosclerosis 222: 541-544. 